DOI https://doi.org/10.24297/jam.v20i.8989

\title{
Coefficient Bounds and Fekete-Szegö inequality for a Certain Families of Bi-Prestarlike Functions Defined by (M,N)-Lucas Polynomials
}

Najah Ali Jiben Al-Ziadi ${ }^{1}$ and Abbas Kareem Wanas ${ }^{2}$

${ }^{1}$ Department of Mathematics, College of Education

University of Al-Qadisiyah, Al Diwaniyah, Al-Qadisiyah, Iraq

najah.ali@qu.edu.iq

${ }^{2}$ Department of Mathematics, College of Science

University of Al-Qadisiyah, Al Diwaniyah, Al-Qadisiyah, Iraq

abbas.kareem.w@qu.edu.iq

\begin{abstract}
:
In the current work, we use the (M,N)-Lucas Polynomials to introduce a new families of holomorphic and bi-Prestarlike functions defined in the unit disk $\mathfrak{O}$ and establish upper bounds for the second and third coefficients of the TaylorMaclaurin series expansions of functions belonging to these families. Also, we debate Fekete-Szegö problem for these families. Further, we point out several certain special cases for our results.
\end{abstract}

Keywords: Bi-Univalent function, Bi-Prestarlike function, (M,N)-Lucas Polynomials, Coefficient bounds, Fekete-Szegö problem, Subordination.

2010 Mathematical Subject Classification: 30C45, 30C50.

\section{Introduction}

Indicate by $\mathfrak{A}$ the collection of functions $\mathfrak{U}$ that are holomorphic in the unit disk $\mathfrak{O}=\{\xi \in \mathbb{C}:|\xi|<1\}$ that have the shape:

$$
\mathfrak{U}(\xi)=\xi+\sum_{n=2}^{\infty} a_{n} \xi^{n}
$$

Further, let $\mathfrak{S}$ stands for the subfamily of the collection $\mathfrak{A}$ consisting of functions in $\mathfrak{O}$ satisfying (1.1) that are univalent in $\mathfrak{O}$. According to "the Koebe one-quarter theorem" (see [12]), each univalent function of this kind has an inverse $\mathfrak{U}^{-1}$ that fulfills

$$
\mathfrak{U}^{-1}(\mathfrak{U}(\xi))=\xi \quad(\xi \in \mathfrak{O})
$$

and

$$
\mathfrak{U}\left(\mathfrak{U}^{-1}(\zeta)\right)=\zeta, \quad\left(|\zeta|<r_{0}(\mathfrak{U}), r_{0}(\mathfrak{U}) \geq \frac{1}{4}\right),
$$

where

$$
\mathfrak{U}^{-1}(\zeta)=\zeta-a_{2} \zeta^{2}+\left(2 a_{2}^{2}-a_{3}\right) \zeta^{3}-\left(5 a_{2}^{3}-5 a_{2} a_{3}+a_{4}\right) \zeta^{4}+\cdots
$$

A function $\mathfrak{U} \in \mathfrak{A}$ is said to be bi-univalent in $\mathfrak{O}$ if both $\mathfrak{U}$ and $\mathfrak{U}^{-1}$ are univalent in $\mathfrak{O}$, let we name by the notation $\mathfrak{E}$ the set of bi-univalent functions in $\mathfrak{O}$ satisfying (1.1). In fact, Srivastava et al. [32] refreshed the study of holomorphic and biunivalent functions in recent years, it was followed by other works as those by Frasin and Aouf [15], Altinkaya and Yalçin 
[5], Güney et al. [16] and others (see, for example [1, 3, 8, 10, 11, 18, 21, 22, 23, 26, 27, 28, 29, 30, 31, 33, 34, 35, 38, 39, 41]). The problem to obtain the general coefficient bounds on the Taylor-Maclaurin coefficients

$$
\left|a_{n}\right| \quad(n \in \mathbb{N} ; n \geq 4)
$$

for functions $\mathfrak{U} \in \mathfrak{E}$ is still not completely addressed for many of the subfamilies of the bi-univalent function class $\mathfrak{E}$. The Fekete-Szegö functional $\left|a_{3}-\mu a_{2}^{2}\right|$ for $\mathfrak{U} \in \mathfrak{S}$ is well known for its rich history in the field of Geometric Function Theory. Its origin was in the disproof by Fekete and Szegö [13] of the Littlewood-Paley conjecture that the coefficients of odd univalent functions are bounded by unity.

A function $\mathfrak{U} \in \mathfrak{A}$ is named starlike of order $\theta(0 \leq \theta<1)$, if

$$
\Re\left\{\frac{\xi \mathfrak{U}^{\prime}(\xi)}{\mathfrak{U}(\xi)}\right\}>\theta, \quad(\xi \in \mathfrak{O}) .
$$

For $\mathfrak{U} \in \mathfrak{A}$ given by (1.1) and $\mathfrak{J} \in \mathfrak{A}$ defined by

$$
\mathfrak{J}(\xi)=\xi+\sum_{n=2}^{\infty} b_{n} \xi^{n}
$$

the "Hadamard product" of $\mathfrak{U}$ and $\mathfrak{J}$ is defined by

$$
(\mathfrak{U} * \mathfrak{J})(\xi)=\xi+\sum_{n=2}^{\infty} a_{n} b_{n} \xi^{n}, \quad(\xi \in \mathfrak{O}) .
$$

Ruscheweyh [25] introduced and studied the family of "prestarlike functions" of order $\theta$, that are the function $\mathfrak{U}$ such as $\mathfrak{U} * I_{\theta}$ is a starlike function of order $\theta$, where

$$
I_{\theta}(\xi)=\frac{\xi}{(1-\xi)^{2(1-\theta)}}, \quad(0 \leq \theta<1, \xi \in \mathfrak{O}) .
$$

The function $I_{\theta}$ can be written in the form:

$$
I_{\theta}(\xi)=\xi+\sum_{n=2}^{\infty} \varrho_{n}(\theta) \xi^{n}
$$

where

$$
\varrho_{n}(\theta)=\frac{\prod_{i=2}^{n}(i-2 \theta)}{(n-1) !}, \quad n \geq 2 .
$$

We note that $\varrho_{n}(\theta)$ is a decreasing function in $\theta$ and satisfies

$$
\lim _{n \rightarrow \infty} \varrho_{n}(\theta)=\left\{\begin{array}{ll}
\infty, & \text { if } \theta<\frac{1}{2} \\
1, & \text { if } \theta=\frac{1}{2} \\
0, & \text { if } \theta>\frac{1}{2}
\end{array} .\right.
$$

With a view to remembering the principle of subordination between holomorphic functions, let the functions $\mathfrak{U}$ and $\mathfrak{J}$ be holomorphic in $\mathfrak{O}$, we name the function $\mathfrak{U}$ is subordinate to $\mathfrak{J}$, if there is a Schwarz function $\hbar$ holomorphic in $\mathfrak{O}$ with

$$
\hbar(0)=0 \quad \text { and } \quad|\hbar(\xi)|<1 \quad(\xi \in \mathfrak{O})
$$

such that

$$
\mathfrak{U}(\xi)=\mathfrak{J}(\hbar(\xi))
$$


This subordination is indicated by

$$
\mathfrak{U} \prec \mathfrak{J} \quad \text { or } \quad \mathfrak{U}(\xi) \prec \mathfrak{J}(\xi) \quad(\xi \in \mathfrak{O}) .
$$

For two polynomials $M(x)$ and $N(x)$ that have real-valued coefficients, the following recurrence relation gives the $(\mathrm{M}, \mathrm{N})$-Lucas Polynomials $L_{M, N, k}(x)$ (see [19]):

$$
L_{M, N, k}(x)=M(x) L_{M, N, k-1}(x)+N(x) L_{M, N, k-2}(x) \quad(k \geq 2),
$$

with

$$
L_{M, N, 0}(x)=2, \quad L_{M, N, 1}(x)=M(x) \quad \text { and } \quad L_{M, N, 2}(x)=M^{2}(x)+2 N(x) .
$$

The function that generates (M,N)-Lucas Polynomial $L_{M, N, k}(x)$ (see [20]) is given by

$$
T_{\left\{L_{M, N, k}(x)\right\}}(\xi)=\sum_{k=2}^{\infty} L_{M, N, k}(x) \xi^{k}=\frac{2-M(x) \xi}{1-M(x) \xi-N(x) \xi^{2}} .
$$

Remark 1.1. For particular choices of $M(x)$ and $N(x)$, the $(M, N)$-Lucas Polynomial $L_{M, N, k}(x)$ leads to various polynomials, among those we list following few here:

(1) $L_{x, 1, k}(x)=: L_{k}(x)$, the Lucas polynomials,

(2) $L_{2 x, 1, k}(x)=: P_{k}(x)$, the Pell-Lucas polynomials,

(3) $L_{1,2 x, k}(x)=: J_{k}(x)$, the Jacobsthal polynomials,

(4) $L_{3 x,-2, k}(x)=: F_{k}(x)$, the Fermat-Lucas polynomials,

(5) $L_{2 x,-1, k}(x)=: T_{k}(x)$, the first kind Chebyshev polynomials.

We also note that the Lucas polynomials and other special polynomials plays an important role in a diversity of disciplines in the mathematical, statistical, physical and engineering sciences. More details associated with these polynomials can be found in $[2,17,37,14,20,40]$.

In recent years, the (M,N)-Lucas Polynomial was presented and investigated analogously by the various penmans (see, for example,[2, 4, 6, 7, 9, 24, 36]).

\section{Main Results}

This section start with defining the families $\mathcal{W} \mathcal{N}_{\mathfrak{E}}(\delta, \lambda, \theta ; x)$ and $\mathcal{W} \mathcal{M}_{\mathfrak{E}}(\tau, \theta ; x)$ as follows:

Definition 2.1. Assume that $\delta \geq 0,0 \leq \lambda \leq 1$ and $0 \leq \theta<1$, a function $\mathfrak{U} \in \mathfrak{E}$ is called in the family $\mathcal{W} \mathcal{N} \mathfrak{E}(\delta, \lambda, \theta ; x)$ if it fulfills the subordinations:

$$
\begin{aligned}
& (1-\delta)\left[(1-\lambda) \frac{\xi\left(\mathfrak{U} * I_{\theta}\right)^{\prime}(\xi)}{\left(\mathfrak{U} * I_{\theta}\right)(\xi)}+\lambda\left(1+\frac{\xi\left(\mathfrak{U} * I_{\theta}\right)^{\prime \prime}(\xi)}{\left(\mathfrak{U} * I_{\theta}\right)^{\prime}(\xi)}\right)\right]+\delta \frac{\lambda \xi^{2}\left(\mathfrak{U} * I_{\theta}\right)^{\prime \prime}(\xi)+\xi\left(\mathfrak{U} * I_{\theta}\right)^{\prime}(\xi)}{\lambda \xi\left(\mathfrak{U} * I_{\theta}\right)^{\prime}(\xi)+(1-\lambda)\left(\mathfrak{U} * I_{\theta}\right)(\xi)} \\
& \prec T_{\left\{L_{M, N, k}(x)\right\}}(\xi)-1
\end{aligned}
$$

and

$$
\begin{aligned}
& (1-\delta)\left[(1-\lambda) \frac{\zeta\left(\mathfrak{J} * I_{\theta}\right)^{\prime}(\zeta)}{\left(\mathfrak{J} * I_{\theta}\right)(\zeta)}+\lambda\left(1+\frac{\zeta\left(\mathfrak{J} * I_{\theta}\right)^{\prime \prime}(\zeta)}{\left(\mathfrak{J} * I_{\theta}\right)^{\prime}(\zeta)}\right)\right]+\delta \frac{\lambda \zeta^{2}\left(\mathfrak{J} * I_{\theta}\right)^{\prime \prime}(\zeta)+\zeta\left(\mathfrak{J} * I_{\theta}\right)^{\prime}(\zeta)}{\lambda \zeta\left(\mathfrak{J} * I_{\theta}\right)^{\prime}(\zeta)+(1-\lambda)\left(\mathfrak{J} * I_{\theta}\right)(\zeta)} \\
& \prec T_{\left\{L_{M, N, k}(x)\right\}}(\zeta)-1,
\end{aligned}
$$

where $\mathfrak{J}=\mathfrak{U}^{-1}$ is given by (1.2). 
In particular, if we choose $\delta=\lambda=0$ and $\theta=\frac{1}{2}$ in Definition 2.1, we have $\mathcal{W} \mathcal{N}_{\mathfrak{E}}\left(0,0, \frac{1}{2} ; x\right) \equiv S_{\mathfrak{E}}(x)$ for the bi-starlike functions that was given by Altinkaya [4] and satisfying the following subordinations:

$$
\frac{\xi \mathfrak{U}^{\prime}(\xi)}{\mathfrak{U}(\xi)} \prec T_{\left\{L_{M, N, k}(x)\right\}}(\xi)-1
$$

and

$$
\frac{\zeta \mathfrak{J}^{\prime}(\zeta)}{\mathfrak{J}(\zeta)} \prec T_{\left\{L_{M, N, k}(x)\right\}}(\zeta)-1
$$

If we choose $\delta=0, \lambda=1$ and $\theta=\frac{1}{2}$ in Definition 2.1, we have $\mathcal{W} \mathcal{N}_{\mathfrak{E}}\left(0,1, \frac{1}{2} ; x\right) \equiv C_{\mathfrak{E}}(x)$ for the bi-convex functions which which was given by Altinkaya [4] and satisfying the following subordinations:

$$
1+\frac{\xi \mathfrak{U}^{\prime \prime}(\xi)}{\mathfrak{U}^{\prime}(\xi)} \prec T_{\left\{L_{M, N, k}(x)\right\}}(\xi)-1
$$

and

$$
1+\frac{\zeta \mathfrak{J}^{\prime \prime}(\zeta)}{\mathfrak{J}^{\prime}(\zeta)} \prec T_{\left\{L_{M, N, k}(x)\right\}}(\zeta)-1 .
$$

Definition 2.2. Assume that $0 \leq \tau \leq 1$ and $0 \leq \theta<1$, a function $f \in \mathfrak{E}$ is called in the family $\mathcal{W} \mathcal{M}_{\mathfrak{E}}(\tau, \theta ; x)$ if it fulfills the subordinations:

$$
\tau \xi\left(\mathfrak{U} * I_{\theta}\right)^{\prime \prime}(\xi)+(2 \tau+1)\left(\mathfrak{U} * I_{\theta}\right)^{\prime}(\xi)-2 \tau \prec T_{\left\{L_{M, N, k}(x)\right\}}(\xi)-1
$$

and

$$
\tau \zeta\left(\mathfrak{J} * I_{\theta}\right)^{\prime \prime}(\zeta)+(2 \tau+1)\left(\mathfrak{J} * I_{\theta}\right)^{\prime}(\zeta)-2 \tau \prec T_{\left\{L_{M, N, k}(x)\right\}}(\zeta)-1
$$

where $\mathfrak{J}=\mathfrak{U}^{-1}$ is given by (1.2).

In particular, if we choose $\tau=0$ and $\theta=\frac{1}{2}$ in Definition 2.2 , we have $\mathcal{W} \mathcal{M}_{\mathfrak{E}}\left(0, \frac{1}{2} ; x\right) \equiv \mathcal{W} \mathcal{M}_{\mathfrak{E}}(x)$ which satisfying the following subordinations:

$$
\mathfrak{U}^{\prime}(\xi) \prec T_{\left\{L_{M, N, k}(x)\right\}}(\xi) \xi-1
$$

and

$$
\mathfrak{J}^{\prime}(\zeta) \prec T_{\left\{L_{M, N, k}(x)\right\}}(\zeta)-1 .
$$

Theorem 2.1. For $\delta \geq 0,0 \leq \lambda \leq 1$ and $0 \leq \theta<1$, let $\mathfrak{U} \in \mathfrak{A}$ belongs to the family $\mathcal{W} \mathcal{N}_{\mathfrak{E}}(\delta, \lambda, \theta ; x)$. Then

$$
\left|a_{2}\right| \leq \frac{|M(x)| \sqrt{|M(x)|}}{\sqrt{2\left|\left[(1-\theta) \Omega(\lambda, \delta, \theta)-2(1-\theta)^{2}(\lambda+1)^{2}\right] M^{2}(x)-4(1-\theta)^{2}(\lambda+1)^{2} N(x)\right|}}
$$

and

$$
\left|a_{3}\right| \leq \frac{M^{2}(x)}{4(1-\theta)^{2}(\lambda+1)^{2}}+\frac{|M(x)|}{2(1-\theta)(3-2 \theta)(2 \lambda+1)},
$$

where

$$
\Omega(\lambda, \delta, \theta)=2 \lambda \delta(1-\theta)(1-\lambda)+2 \theta \lambda+1 .
$$

Proof. Suppose that $\mathfrak{U} \in \mathcal{W} \mathcal{N}_{\mathfrak{E}}(\delta, \lambda, \theta ; x)$. Then there exists two holomorphic functions $\phi, \psi: \mathfrak{O} \longrightarrow \mathfrak{O}$ given by

$$
\phi(\xi)=r_{1} \xi+r_{2} \xi^{2}+r_{3} \xi^{3}+\cdots \quad(\xi \in \mathfrak{O})
$$


and

$$
\psi(\zeta)=s_{1} \zeta+s_{2} \zeta^{2}+s_{3} \zeta^{3}+\cdots \quad(\zeta \in \mathfrak{O}),
$$

with $\phi(0)=\psi(0)=0,|\phi(\xi)|<1,|\psi(\zeta)|<1, \xi, \zeta \in \mathfrak{O}$ such that

$$
\begin{aligned}
& (1-\delta)\left[(1-\lambda) \frac{\xi\left(\mathfrak{U} * I_{\theta}\right)^{\prime}(\xi)}{\left(\mathfrak{U} * I_{\theta}\right)(\xi)}+\lambda\left(1+\frac{\xi\left(\mathfrak{U} * I_{\theta}\right)^{\prime \prime}(\xi)}{\left(\mathfrak{U} * I_{\theta}\right)^{\prime}(\xi)}\right)\right]+\delta \frac{\lambda \xi^{2}\left(\mathfrak{U} * I_{\theta}\right)^{\prime \prime}(\xi)+\xi\left(\mathfrak{U} * I_{\theta}\right)^{\prime}(\xi)}{\lambda \xi\left(\mathfrak{U} * I_{\theta}\right)^{\prime}(\xi)+(1-\lambda)\left(\mathfrak{U} * I_{\theta}\right)(\xi)} \\
& =-1+L_{M, N, 0}(x)+L_{M, N, 1}(x) \phi(\xi)+L_{M, N, 2}(x) \phi^{2}(\xi)+\cdots
\end{aligned}
$$

and

$$
\begin{aligned}
& (1-\delta)\left[(1-\lambda) \frac{\zeta\left(\mathfrak{J} * I_{\theta}\right)^{\prime}(\zeta)}{\left(\mathfrak{J} * I_{\theta}\right)(\zeta)}+\lambda\left(1+\frac{\zeta\left(\mathfrak{J} * I_{\theta}\right)^{\prime \prime}(\zeta)}{\left(\mathfrak{J} * I_{\theta}\right)^{\prime}(\zeta)}\right)\right]+\delta \frac{\lambda \zeta^{2}\left(\mathfrak{J} * I_{\theta}\right)^{\prime \prime}(\zeta)+\zeta\left(\mathfrak{J} * I_{\theta}\right)^{\prime}(\zeta)}{\lambda \zeta\left(\mathfrak{J} * I_{\theta}\right)^{\prime}(\zeta)+(1-\lambda)\left(\mathfrak{J} * I_{\theta}\right)(\zeta)} \\
& =-1+L_{M, N, 0}(x)+L_{M, N, 1}(x) \psi(\zeta)+L_{M, N, 2}(x) \psi^{2}(\zeta)+\cdots
\end{aligned}
$$

Combining (2.2), (2.3), (2.4) and (2.5), yield

$$
\begin{aligned}
& (1-\delta)\left[(1-\lambda) \frac{\xi\left(\mathfrak{U} * I_{\theta}\right)^{\prime}(\xi)}{\left(\mathfrak{U} * I_{\theta}\right)(\xi)}+\lambda\left(1+\frac{\xi\left(\mathfrak{U} * I_{\theta}\right)^{\prime \prime}(\xi)}{\left(\mathfrak{U} * I_{\theta}\right)^{\prime}(\xi)}\right)\right]+\delta \frac{\lambda \xi^{2}\left(\mathfrak{U} * I_{\theta}\right)^{\prime \prime}(\xi)+\xi\left(\mathfrak{U} * I_{\theta}\right)^{\prime}(\xi)}{\lambda \xi\left(\mathfrak{U} * I_{\theta}\right)^{\prime}(\xi)+(1-\lambda)\left(\mathfrak{U} * I_{\theta}\right)(\xi)} \\
& =1+L_{M, N, 1}(x) r_{1} \xi+\left[L_{M, N, 1}(x) r_{2}+L_{M, N, 2}(x) r_{1}^{2}\right] \xi^{2}+\cdots
\end{aligned}
$$

and

$$
\begin{aligned}
& (1-\delta)\left[(1-\lambda) \frac{\zeta\left(\mathfrak{J} * I_{\theta}\right)^{\prime}(\zeta)}{\left(\mathfrak{J} * I_{\theta}\right)(\zeta)}+\lambda\left(1+\frac{\zeta\left(\mathfrak{J} * I_{\theta}\right)^{\prime \prime}(\zeta)}{\left(\mathfrak{J} * I_{\theta}\right)^{\prime}(\zeta)}\right)\right]+\delta \frac{\lambda \zeta^{2}\left(\mathfrak{J} * I_{\theta}\right)^{\prime \prime}(\zeta)+\zeta\left(\mathfrak{J} * I_{\theta}\right)^{\prime}(\zeta)}{\lambda \zeta\left(\mathfrak{J} * I_{\theta}\right)^{\prime}(\zeta)+(1-\lambda)\left(\mathfrak{J} * I_{\theta}\right)(\zeta)} \\
& =1+L_{M, N, 1}(x) s_{1} \zeta+\left[L_{M, N, 1}(x) s_{2}+L_{M, N, 2}(x) s_{1}^{2}\right] \zeta^{2}+\cdots
\end{aligned}
$$

It is quite well-known that if $|\phi(\xi)|<1$ and $|\psi(\zeta)|<1, \xi, \zeta \in \mathfrak{O}$, we get

$$
\left|r_{j}\right| \leq 1 \quad \text { and } \quad\left|s_{j}\right| \leq 1(j \in \mathbb{N})
$$

In the light of (2.6) and (2.7), after simplifying, we find that

$$
\begin{gathered}
2(1-\theta)(\lambda+1) a_{2}=L_{M, N, 1}(x) r_{1}, \\
2(1-\theta)(3-2 \theta)(2 \lambda+1) a_{3}-4(1-\theta)^{2}(\lambda \delta(\lambda-1)+3 \lambda+1) a_{2}^{2}=L_{M, N, 1}(x) r_{2}+L_{M, N, 2}(x) r_{1}^{2}, \\
-2(1-\theta)(\lambda+1) a_{2}=L_{M, N, 1}(x) s_{1}
\end{gathered}
$$

and

$$
\begin{aligned}
2(1-\theta)(3-2 \theta)(2 \lambda+1)\left(2 a_{2}^{2}-a_{3}\right) & -4(1-\theta)^{2}(\lambda \delta(\lambda-1)+3 \lambda+1) a_{2}^{2} \\
& =L_{M, N, 1}(x) s_{2}+L_{M, N, 2}(x) s_{1}^{2} .
\end{aligned}
$$

It follows from (2.9) and (2.11) that

$$
r_{1}=-s_{1}
$$

and

$$
8(1-\theta)^{2}(\lambda+1)^{2} a_{2}^{2}=L_{M, N, 1}^{2}(x)\left(r_{1}^{2}+s_{1}^{2}\right) .
$$

If we add (2.10) to (2.12), we obtain

$$
4(1-\theta)[2 \lambda \delta(1-\theta)(1-\lambda)+2 \theta \lambda+1] a_{2}^{2}=L_{M, N, 1}(x)\left(r_{2}+s_{2}\right)+L_{M, N, 2}(x)\left(r_{1}^{2}+s_{1}^{2}\right) .
$$


By substitute the value of $r_{1}^{2}+s_{1}^{2}$ from (2.14) in the right hand side of (2.15), we conclude that

$$
\left[4(1-\theta) \Omega(\lambda, \delta, \theta)-\frac{8 L_{M, N, 2}(x)}{L_{M, N, 1}^{2}(x)}(1-\theta)^{2}(\lambda+1)^{2}\right] a_{2}^{2}=L_{M, N, 1}(x)\left(r_{2}+s_{2}\right),
$$

where $\Omega(\lambda, \delta, \theta)$ is given by $(2.1)$.

Moreover computations using (1.3), (2.8) and (2.16), we find that

$$
\left|a_{2}\right| \leq \frac{|M(x)| \sqrt{|M(x)|}}{\sqrt{2\left|\left[(1-\theta) \Omega(\lambda, \delta, \theta)-2(1-\theta)^{2}(\lambda+1)^{2}\right] M^{2}(x)-4(1-\theta)^{2}(\lambda+1)^{2} N(x)\right|}} .
$$

Next, if we subtract (2.12) from (2.10), we can easily see that

$$
4(1-\theta)(3-2 \theta)(2 \lambda+1)\left(a_{3}-a_{2}^{2}\right)=L_{M, N, 1}(x)\left(r_{2}-s_{2}\right)+L_{M, N, 2}(x)\left(r_{1}^{2}-s_{1}^{2}\right) .
$$

In view of (2.13) and (2.14), we get from (2.17)

$$
a_{3}=\frac{L_{M, N, 1}^{2}(x)}{8(1-\theta)^{2}(\lambda+1)^{2}}\left(r_{1}^{2}+s_{1}^{2}\right)+\frac{L_{M, N, 1}(x)}{4(1-\theta)(3-2 \theta)(2 \lambda+1)}\left(r_{2}-s_{2}\right) .
$$

Thus applying (1.3), we conclude that

$$
\left|a_{3}\right| \leq \frac{M^{2}(x)}{4(1-\theta)^{2}(\lambda+1)^{2}}+\frac{|M(x)|}{2(1-\theta)(3-2 \theta)(2 \lambda+1)} .
$$

Putting $\delta=\lambda=0$ and $\theta=\frac{1}{2}$ in Theorem 2.1, we deduce the next outcome:

Corollary 2.1. [4] If $\mathfrak{U}$ belongs to the family $S_{\mathfrak{E}}(x)$, then

$$
\left|a_{2}\right| \leq|M(x)| \sqrt{\left|\frac{M(x)}{2 N(x)}\right|}
$$

and

$$
\left|a_{3}\right| \leq M^{2}(x)+\frac{|M(x)|}{2}
$$

Putting $\delta=0, \lambda=1$ and $\theta=\frac{1}{2}$ in Theorem 2.1, we deduce the next outcome:

Corollary 2.2. [4] If $\mathfrak{U}$ belongs to the family $C_{\mathfrak{E}}(x)$, then

$$
\left|a_{2}\right| \leq \frac{|M(x)| \sqrt{|M(x)|}}{\sqrt{2\left|M^{2}(x)+4 N(x)\right|}}
$$

and

$$
\left|a_{3}\right| \leq \frac{M^{2}(x)}{4}+\frac{|M(x)|}{6}
$$

Theorem 2.2. For $0 \leq \tau \leq 1$ and $0 \leq \theta<1$, let $\mathfrak{U} \in \mathfrak{A}$ belongs to the family $\mathcal{W} \mathcal{M}_{\mathfrak{E}}(\tau, \theta ; x)$. Then

$$
\left|a_{2}\right| \leq \frac{|M(x)| \sqrt{|M(x)|}}{\sqrt{\left|\left[3(1-\theta)(3-2 \theta)(4 \tau+1)-4(1-\theta)^{2}(7 \tau+3)^{2}\right] M^{2}(x)-8(1-\theta)^{2}(7 \tau+3)^{2} N(x)\right|}}
$$

and

$$
\left|a_{3}\right| \leq \frac{M^{2}(x)}{4(1-\theta)^{2}(7 \tau+3)^{2}}+\frac{|M(x)|}{3(1-\theta)(3-2 \theta)(4 \tau+1)}
$$


Proof. Suppose that $\mathfrak{U} \in \mathcal{W} \mathcal{M}_{\mathfrak{E}}(\tau, \theta ; x)$. Then there exists two holomorphic functions $\phi, \psi: \mathfrak{O} \longrightarrow \mathfrak{O}$ such that

$$
\begin{aligned}
& \tau \xi\left(\mathfrak{U} * I_{\theta}\right)^{\prime \prime}(\xi)+(2 \tau+1)\left(\mathfrak{U} * I_{\theta}\right)^{\prime}(\xi)-2 \tau \\
& =-1+L_{M, N, 0}(x)+L_{M, N, 1}(x) \phi(\xi)+L_{M, N, 2}(x) \phi^{2}(\xi)+\cdots
\end{aligned}
$$

and

$$
\begin{aligned}
& \tau \zeta\left(\mathfrak{J} * I_{\theta}\right)^{\prime \prime}(\zeta)+(2 \tau+1)\left(\mathfrak{J} * I_{\theta}\right)^{\prime}(\zeta)-2 \tau \\
& =-1+L_{M, N, 0}(x)+L_{M, N, 1}(x) \psi(\zeta)+L_{M, N, 2}(x) \psi^{2}(\zeta)+\cdots
\end{aligned}
$$

where $\phi$ and $\psi$ have the forms (2.2) and (2.3). Combining (2.18) and (2.19), yield

$$
\begin{aligned}
& \tau \xi\left(\mathfrak{U} * I_{\theta}\right)^{\prime \prime}(\xi)+(2 \tau+1)\left(\mathfrak{U} * I_{\theta}\right)^{\prime}(\xi)-2 \tau \\
& =1+L_{M, N, 1}(x) r_{1} \xi+\left[L_{M, N, 1}(x) r_{2}+L_{M, N, 2}(x) r_{1}^{2}\right] \xi^{2}+\cdots
\end{aligned}
$$

and

$$
\begin{aligned}
& \tau \zeta\left(\mathfrak{J} * I_{\theta}\right)^{\prime \prime}(\zeta)+(2 \tau+1)\left(\mathfrak{J} * I_{\theta}\right)^{\prime}(\zeta)-2 \tau \\
& =1+L_{M, N, 1}(x) s_{1} \zeta+\left[L_{M, N, 1}(x) s_{2}+L_{M, N, 2}(x) s_{1}^{2}\right] \zeta^{2}+\cdots
\end{aligned}
$$

In the light of (2.20) and (2.21), after simplifying, we find that

$$
\begin{gathered}
2(1-\theta)(7 \tau+3) a_{2}=L_{M, N, 1}(x) r_{1}, \\
3(1-\theta)(3-2 \theta)(4 \tau+1) a_{3}=L_{M, N, 1}(x) r_{2}+L_{M, N, 2}(x) r_{1}^{2}, \\
-2(1-\theta)(7 \tau+3) a_{2}=L_{M, N, 1}(x) s_{1}
\end{gathered}
$$

and

$$
3(1-\theta)(3-2 \theta)(4 \tau+1)\left(2 a_{2}^{2}-a_{3}\right)=L_{M, N, 1}(x) s_{2}+L_{M, N, 2}(x) s_{1}^{2} .
$$

It follows from (2.22) and (2.24) that

$$
r_{1}=-s_{1}
$$

and

$$
8(1-\theta)^{2}(7 \tau+3)^{2} a_{2}^{2}=L_{M, N, 1}^{2}(x)\left(r_{1}^{2}+s_{1}^{2}\right) .
$$

If we add (2.23) to (2.25), we obtain

$$
6(1-\theta)(3-2 \theta)(4 \tau+1) a_{2}^{2}=L_{M, N, 1}(x)\left(r_{2}+s_{2}\right)+L_{M, N, 2}(x)\left(r_{1}^{2}+s_{1}^{2}\right) .
$$

By substitute the value of $r_{1}^{2}+s_{1}^{2}$ from (2.27) in the right hand side of (2.28), we conclude that

$$
\left[6(1-\theta)(3-2 \theta)(4 \tau+1)-\frac{8 L_{M, N, 2}(x)}{L_{M, N, 1}^{2}(x)}(1-\theta)^{2}(7 \tau+3)^{2}\right] a_{2}^{2}=L_{M, N, 1}(x)\left(r_{2}+s_{2}\right)
$$

Moreover computations using (1.3), (2.8) and (2.29), we find that

$$
\left|a_{2}\right| \leq \frac{|M(x)| \sqrt{|M(x)|}}{\sqrt{\left|\left[3(1-\theta)(3-2 \theta)(4 \tau+1)-4(1-\theta)^{2}(7 \tau+3)^{2}\right] M^{2}(x)-8(1-\theta)^{2}(7 \tau+3)^{2} N(x)\right|}}
$$


Next, if we subtract (2.25) from (2.23), we can easily see that

$$
6(1-\theta)(3-2 \theta)(4 \tau+1)\left(a_{3}-a_{2}^{2}\right)=L_{M, N, 1}(x)\left(r_{2}-s_{2}\right)+L_{M, N, 2}(x)\left(r_{1}^{2}-s_{1}^{2}\right) .
$$

In view of (2.26) and (2.27), we get from (2.30)

$$
a_{3}=\frac{L_{M, N, 1}^{2}(x)}{8(1-\theta)^{2}(7 \tau+3)^{2}}\left(r_{1}^{2}+s_{1}^{2}\right)+\frac{L_{M, N, 1}(x)}{6(1-\theta)(3-2 \theta)(4 \tau+1)}\left(r_{2}-s_{2}\right) .
$$

Thus applying (1.3), we conclude that

$$
\left|a_{3}\right| \leq \frac{M^{2}(x)}{4(1-\theta)^{2}(7 \tau+3)^{2}}+\frac{|M(x)|}{3(1-\theta)(3-2 \theta)(4 \tau+1)}
$$

Putting $\tau=0$ and $\theta=\frac{1}{2}$ in Theorem 2.2, we deduce the next outcome:

Corollary 2.3. If $\mathfrak{U}$ belongs to the family $\mathcal{W} \mathcal{M}_{\mathfrak{E}}(x)$, then

$$
\left|a_{2}\right| \leq \frac{|M(x)| \sqrt{|M(x)|}}{\sqrt{6\left|M^{2}(x)+3 N(x)\right|}}
$$

and

$$
\left|a_{3}\right| \leq \frac{M^{2}(x)}{9}+\frac{|M(x)|}{3}
$$

In the following theorems, we introduce the Fekete-Szegö Problem of the families $\mathcal{W} \mathcal{N}_{\mathfrak{E}}(\delta, \lambda, \theta ; x)$ and $\mathcal{W} \mathcal{M}_{\mathfrak{E}}(\tau, \theta ; x)$.

Theorem 2.3. For $\delta \geq 0,0 \leq \lambda \leq 1,0 \leq \theta<1$ and $\rho \in \mathbb{R}$, let $\mathfrak{U} \in \mathfrak{A}$ belongs to the family $\mathcal{W} \mathcal{N} \mathfrak{E}(\delta, \lambda, \theta ; x)$. Then

$$
\left|a_{3}-\rho a_{2}^{2}\right| \leq\left\{\begin{array}{r}
\frac{|M(x)|}{2(1-\theta)(3-2 \theta)(2 \lambda+1)} ; \\
\text { for }|\rho-1| \leq \frac{\left|(1-\theta) \Omega(\lambda, \delta, \theta)-2(1-\theta)^{2}(\lambda+1)^{2}-\frac{4(1-\theta)^{2}(\lambda+1)^{2} N(x)}{M^{2}(x)}\right|}{(1-\theta)(3-2 \theta)(2 \lambda+1)}, \\
\frac{|M(x)|^{3}|\rho-1|}{2\left|\left[(1-\theta) \Omega(\lambda, \delta, \theta)-2(1-\theta)^{2}(\lambda+1)^{2}\right] M^{2}(x)-4(1-\theta)^{2}(\lambda+1)^{2} N(x)\right|} ; \\
\text { for }|\rho-1| \geq \frac{\left|(1-\theta) \Omega(\lambda, \delta, \theta)-2(1-\theta)^{2}(\lambda+1)^{2}-\frac{4(1-\theta)^{2}(\lambda+1)^{2} N(x)}{M^{2}(x)}\right|}{(1-\theta)(3-2 \theta)(2 \lambda+1)}
\end{array}\right.
$$

where $\Omega(\lambda, \delta, \theta)$ is given by (2.1).

Proof. By making use of (2.16) and (2.17), we conclude that

$$
\begin{aligned}
a_{3}-\rho a_{2}^{2} & =\frac{L_{M, N, 1}^{3}(x)\left(r_{2}+s_{2}\right)(1-\rho)}{4\left[L_{M, N, 1}^{2}(x)(1-\theta) \Omega(\lambda, \delta, \theta)-2 L_{M, N, 2}(x)(1-\theta)^{2}(\lambda+1)^{2}\right]} \\
& +\frac{L_{M, N, 1}(x)\left(r_{2}-s_{2}\right)}{4(1-\theta)(3-2 \theta)(2 \lambda+1)} \\
& =\frac{L_{M, N, 1}(x)}{4}\left[\left(\varphi(\rho ; x)+\frac{1}{(1-\theta)(3-2 \theta)(2 \lambda+1)}\right) r_{2}\right. \\
& \left.+\left(\varphi(\rho ; x)-\frac{1}{(1-\theta)(3-2 \theta)(2 \lambda+1)}\right) s_{2}\right],
\end{aligned}
$$


where

$$
\varphi(\rho ; x)=\frac{L_{M, N, 1}^{2}(x)(1-\rho)}{L_{M, N, 1}^{2}(x)(1-\theta) \Omega(\lambda, \delta, \theta)-2 L_{M, N, 2}(x)(1-\theta)^{2}(\lambda+1)^{2}} .
$$

According to (1.3), we find that

$$
\left|a_{3}-\rho a_{2}^{2}\right| \leq\left\{\begin{array}{lr}
\frac{|M(x)|}{2(1-\theta)(3-2 \theta)(2 \lambda+1)}, & 0 \leq|\varphi(\rho ; x)| \leq \frac{1}{(1-\theta)(3-2 \theta)(2 \lambda+1)} \\
\frac{1}{2}|M(x)||\varphi(\rho ; x)|, & |\varphi(\rho ; x)| \geq \frac{1}{(1-\theta)(3-2 \theta)(2 \lambda+1)}
\end{array}\right.
$$

After some computations, we obtain

$$
\left|a_{3}-\rho a_{2}^{2}\right| \leq\left\{\begin{array}{c}
\begin{array}{c}
\frac{|M(x)|}{2(1-\theta)(3-2 \theta)(2 \lambda+1)} ; \\
f o r|\rho-1| \leq \\
\frac{\left|(1-\theta) \Omega(\lambda, \delta, \theta)-2(1-\theta)^{2}(\lambda+1)^{2}-\frac{4(1-\theta)^{2}(\lambda+1)^{2} N(x)}{M^{2}(x)}\right|}{(1-\theta)(3-2 \theta)(2 \lambda+1)} \\
\frac{|M(x)|^{3}|\rho-1|}{2\left|\left[(1-\theta) \Omega(\lambda, \delta, \theta)-2(1-\theta)^{2}(\lambda+1)^{2}\right] M^{2}(x)-4(1-\theta)^{2}(\lambda+1)^{2} N(x)\right|}
\end{array} \\
\text { for }|\rho-1| \geq \frac{\left|(1-\theta) \Omega(\lambda, \delta, \theta)-2(1-\theta)^{2}(\lambda+1)^{2}-\frac{4(1-\theta)^{2}(\lambda+1)^{2} N(x)}{M^{2}(x)}\right|}{(1-\theta)(3-2 \theta)(2 \lambda+1)}
\end{array}\right.
$$

Putting $\delta=\lambda=0$ and $\theta=\frac{1}{2}$ in Theorem 2.3, we deduce the next outcome:

Corollary 2.4. [4] If $\mathfrak{U}$ belongs to the family $S_{\mathfrak{E}}(x)$, then

$$
\left|a_{3}-\rho a_{2}^{2}\right| \leq \begin{cases}\frac{|M(x)|}{2} ; & \text { for }|\rho-1| \leq \frac{|N(x)|}{M^{2}(x)} \\ \frac{|M(x)|^{3}|\rho-1|}{2|N(x)|} ; & \text { for }|\rho-1| \geq \frac{|N(x)|}{M^{2}(x)}\end{cases}
$$

Putting $\delta=\lambda=0$ and $\theta=\frac{1}{2}$ in Theorem 2.3, we deduce the next outcome:

Corollary 2.5. [4] If $\mathfrak{U}$ belongs to the family $C_{\mathfrak{E}}(x)$, then

$$
\left|a_{3}-\rho a_{2}^{2}\right| \leq\left\{\begin{array}{l}
\frac{|M(x)|}{6} ; \quad \text { for }|\rho-1| \leq \frac{\left|M^{2}(x)+4 N(x)\right|}{3 M^{2}(x)}, \\
\frac{|M(x)|^{3}|\rho-1|}{2\left|M^{2}(x)+4 N(x)\right|} ; \quad \text { for }|\rho-1| \geq \frac{\left|M^{2}(x)+4 N(x)\right|}{3 M^{2}(x)} .
\end{array}\right.
$$

Putting $\rho=1$ in Theorem 2.3, we deduce the next outcome:

Corollary 2.6. If $\mathfrak{U}$ belongs to the family $\mathcal{W} \mathcal{N}_{\mathfrak{E}}(\delta, \lambda, \theta ; x)$, then

$$
\left|a_{3}-a_{2}^{2}\right| \leq \frac{|M(x)|}{2(1-\theta)(3-2 \theta)(2 \lambda+1)} .
$$

Putting $\rho=1$ in Corollary 2.4, we deduce the next outcome: 
Corollary 2.7. [4] If $\mathfrak{U}$ belongs to the family $S_{\mathfrak{E}}(x)$, then

$$
\left|a_{3}-a_{2}^{2}\right| \leq \frac{|M(x)|}{2} .
$$

Putting $\rho=1$ in Corollary 2.5, we deduce the next outcome:

Corollary 2.8. [4] If $\mathfrak{U}$ belongs to the family $C_{\mathfrak{E}}(x)$, then

$$
\left|a_{3}-a_{2}^{2}\right| \leq \frac{|M(x)|}{6}
$$

Theorem 2.4. For $0 \leq \tau \leq 1,0 \leq \theta<1$ and $\rho \in \mathbb{R}$, let $\mathfrak{U} \in \mathfrak{A}$ belongs to the family $\mathcal{W} \mathcal{M} \mathfrak{E}(\tau, \theta ; x)$. Then

$$
\left|a_{3}-\rho a_{2}^{2}\right| \leq\left\{\begin{array}{r}
\frac{|M(x)|}{3(1-\theta)(3-2 \theta)(4 \tau+1)} ; \\
\text { for }|\rho-1| \leq\left|1-\frac{\frac{4}{3}\left[(1-\theta)^{2}(7 \tau+3)^{2}+\frac{2(1-\theta)^{2}(7 \tau+3)^{2} N(x)}{M^{2}(x)}\right]}{(1-\theta)(3-2 \theta)(4 \tau+1)}\right|
\end{array} \mid\right.
$$

Proof. By making use of (2.29) and (2.30), we conclude that

$$
\begin{aligned}
a_{3}-\rho a_{2}^{2} & =\frac{L_{M, N, 1}^{3}(x)\left(r_{2}+s_{2}\right)(1-\rho)}{2\left[3 L_{M, N, 1}^{2}(x)(1-\theta)(3-2 \theta)(4 \tau+1)-4 L_{M, N, 2}(x)(1-\theta)^{2}(7 \tau+3)^{2}\right]} \\
& +\frac{L_{M, N, 1}(x)\left(r_{2}-s_{2}\right)}{6(1-\theta)(3-2 \theta)(4 \tau+1)} \\
& =\frac{L_{M, N, 1}(x)}{2}\left[\left(\psi(\rho ; x)+\frac{1}{3(1-\theta)(3-2 \theta)(4 \tau+1)}\right) r_{2}\right. \\
& \left.+\left(\psi(\rho ; x)-\frac{1}{3(1-\theta)(3-2 \theta)(4 \tau+1)}\right) s_{2}\right]
\end{aligned}
$$

where

$$
\psi(\rho ; x)=\frac{L_{M, N, 1}^{2}(x)(1-\rho)}{3 L_{M, N, 1}^{2}(x)(1-\theta)(3-2 \theta)(4 \tau+1)-4 L_{M, N, 2}(x)(1-\theta)^{2}(7 \tau+3)^{2}} .
$$

According to (1.3), we find that

$$
\left|a_{3}-\rho a_{2}^{2}\right| \leq\left\{\begin{array}{lr}
\frac{|M(x)|}{3(1-\theta)(3-2 \theta)(4 \tau+1)}, & 0 \leq|\psi(\rho ; x)| \leq \frac{1}{3(1-\theta)(3-2 \theta)(4 \tau+1)} \\
|M(x)||\psi(\rho ; x)|, & |\psi(\rho ; x)| \geq \frac{1}{3(1-\theta)(3-2 \theta)(4 \tau+1)} .
\end{array}\right.
$$


After some computations, we obtain

$$
\left|a_{3}-\rho a_{2}^{2}\right| \leq\left\{\begin{array}{r}
\frac{|M(x)|}{3(1-\theta)(3-2 \theta)(4 \tau+1)} ; \\
\text { for }|\rho-1| \leq\left|1-\frac{\frac{4}{3}\left[(1-\theta)^{2}(7 \tau+3)^{2}+\frac{2(1-\theta)^{2}(7 \tau+3)^{2} N(x)}{M^{2}(x)}\right]}{(1-\theta)(3-2 \theta)(4 \tau+1)}\right|
\end{array} \mid\right.
$$

Putting $\tau=0$ and $\theta=\frac{1}{2}$ in Theorem 2.4, we deduce the next outcome:

Corollary 2.9. If $\mathfrak{U}$ belongs to the family $\mathcal{W} \mathcal{M}_{\mathfrak{E}}(x)$, then

$$
\left|a_{3}-\rho a_{2}^{2}\right| \leq\left\{\begin{array}{l}
\frac{|M(x)|}{3} ; \quad \text { for }|\rho-1| \leq 2\left|1+3 \frac{N(x)}{M^{2}(x)}\right| \\
\frac{|M(x)|^{3}|\rho-1|}{6\left|M^{2}(x)+3 N(x)\right|} ; \quad \text { for }|\rho-1| \geq 2\left|1+3 \frac{N(x)}{M^{2}(x)}\right| .
\end{array}\right.
$$

Putting $\rho=1$ in Theorem 2.4, we deduce the next outcome:

Corollary 2.10. If $\mathfrak{U}$ belongs to the family $\mathcal{W} \mathcal{M}_{\mathfrak{E}}(\tau, \theta ; x)$, then

$$
\left|a_{3}-a_{2}^{2}\right| \leq \frac{|M(x)|}{3(1-\theta)(3-2 \theta)(4 \tau+1)} .
$$

Putting $\rho=1$ in Corollary 2.9, we deduce the next outcome:

Corollary 2.11. If $\mathfrak{U}$ belongs to the family $\mathcal{W} \mathcal{M}_{\mathfrak{E}}(x)$, then

$$
\left|a_{3}-a_{2}^{2}\right| \leq \frac{|M(x)|}{3}
$$

\section{References}

[1] C. Abirami, N. Magesh and J. Yamini, Initial bounds for certain classes of bi-univalent functions defined by Horadam polynomials, Abstr. Appl. Anal., 2020, Art. ID 7391058, (2020), 1-8.

[2] A. Akgül, (P,Q)-Lucas polynomial coefficient inequalities of the bi-univalent function class, Turkish Journal of Mathematics, 43(2019), 2170-2176.

[3] A. G. Al-Amoush, Certain subclasses of bi-univalent functions involving the Poisson distribution associated with Horadam polynomials, Malaya J. Mat., 7(2019), 618-624. 
[4] S. Altinkaya, Inclusion properties of Lucas polynomials for bi-univalent functions introduced through the $q$-analogue of the Noor integral operator, Turkish J. Math., 43(2019), 620-629.

[5] S. Altinkaya and S. Yalçin, Coefficient estimates for two new subclasses of bi-univalent functions with respect to symmetric points, J. Funct. Spaces, 2015, Art. ID 145242, (2015), 1-5.

[6] S. Altinkaya and S. Yalçin, On the (p,q)-Lucas polynomial coefficient bounds of the bi-univalent function class $\sigma$, Boletínde la Sociedad Matemática Mexicana, 25(2019), 567-575.

[7] S. Altinkaya and S. Yalçin, (p,q)-Lucas polynomials and their applications to bi-univalent functions, Proyecciones, $39(5)(2019), 1093-1105$.

[8] A. Amourah, Initial bounds for analytic and bi-univalent functions by means of (p,q)-Chebyshev polynomials defined by differential operator, General Letters in Mathematics, 7 (2019), 45-51.

[9] A. Amourah, B. A. Frasin, G. Murugusundaramoorthy and T. Al-Hawary, Bi-Bazilevič functions of order $\vartheta+i \delta$ associated with (p,q)-Lucas polynomials, AIMS Mathematics, 6(5)(2021), 4296-4305.

[10] S. Bulut, Faber polynomial coefficient estimates for a subclass of analytic bi-univalent functions, Filomat, 30(2016), $1567-1575$.

[11] M. Caglar, E. Deniz and H. M. Srivastava, Second Hankel determinant for certain subclasses of bi-univalent functions, Turkish J. Math., 41(2017), 694-706.

[12] P. L. Duren, Univalent Functions, Grundlehren der Mathematischen Wissenschaften, Band 259, Springer Verlag, New York, Berlin, Heidelberg and Tokyo, 1983.

[13] M. Fekete and G. Szegö, Eine bemerkung uber ungerade schlichte funktionen, J. London Math. Soc., 2(1933), $85-89$.

[14] P. Filipponi and AF. Horadam, Derivative sequences of Fibonacci and Lucas polynomials, Applications of Fibonacci Numbers, 4(1991), 99-108.

[15] B. A. Frasin and M. K. Aouf, New subclasses of bi-univalent functions, Appl. Math. Lett., 24(2011), 1569-1573.

[16] H. Ö. Güney, G. Murugusundaramoorthy and J. Sokół, Subclasses of bi-univalent functions related to shell-like curves connected with Fibonacci numbers, Acta Univ. Sapient. Math., 10(2018), 70-84.

[17] A. F. Horadam and J. M. Mahon, Pell and Pell-Lucas polynomials, The Fibonacci Quarterly, 23(1985), 7-20.

[18] S. Joshi, S. Joshi and H. Pawar, On some subclasses of bi-univalent functions associated with pseudo-starlike functions, J. Egyptian Math. Soc., 24(2016), 522-525.

[19] GY. Lee and M. Aşci, Some properties of the (p,q)-Fibonacci and (p,q)-Lucas polynomials, J. Appl. Math., $2012(2012), 1-18$.

[20] A. Lupas, A guide of Fibonacci and Lucas polynomials, Octagon Math. Mag., 7(1999), 2-12.

[21] N. Magesh and S. Bulut, Chebyshev polynomial coefficient estimates for a class of analytic bi-univalent functions related to pseudo-starlike functions, Afr. Mat., 29(2018), 203-209. 
[22] E. P. Mazi and T. O. Opoola, On some subclasses of bi-univalent functions associating pseudo-starlike functions with Sakaguchi type functions, General Mathematics, 25(2017), 85-95.

[23] G. Murugusundaramoorthy and S. Yalçin, On the $\lambda$-Psedo-bi-starlike functions related to (p,q)-Lucas polynomial, Libertas Mathematica (newseries), 39(2019), 79-88.

[24] H. Orhan and H. Arikan, (P,Q)-Lucas polynomial coefficient inequalities of bi-univalent functions defined by the combination of both operators of Al-Aboudi and Ruscheweyh, Afr. Mat. (2020). https://doi.org/10.1007/s13370020-00847-5

[25] S. Ruscheweyh, Linear operators between classes of prestarlike functions, Comment. Math. Helv., 52(4), (1977), 497-509.

[26] H. M. Srivastava and D. Bansal, Coefficient estimates for a subclass of analytic and bi-univalent functions, J. Egyptian Math. Soc., 23(2015), 242-246.

[27] H. M. Srivastava, S. Bulut, M. Caglar and N. Yagmur, Coefficient estimates for a general subclass of analytic and bi-univalent functions, Filomat, 27(5)(2013), 831-842.

[28] H. M. Srivastava, S. S. Eker and R. M. Ali, Coefficient bounds for a certain class of analytic and bi-univalent functions, Filomat, 29(2015), 1839-1845.

[29] H. M. Srivastava, S. S. Eker, S. G. Hamidi and J. M. Jahangiri, Faber polynomial coefficient estimates for bi-univalent functions defined by the Tremblay fractional derivative operator, Bull. Iranian Math. Soc., 44(1)(2018), 149-157.

[30] H. M. Srivastava, S. Gaboury and F. Ghanim, Coefficient estimates for some general subclasses of analytic and bi-univalent functions, Afr. Mat., 28(2017), 693-706.

[31] H. M. Srivastava, S. Gaboury and F. Ghanim, Coefficient estimates for a general subclass of analytic and biunivalent functions of the Ma-Minda type, Rev. Real Acad. Cienc. Exactas Fís. Natur. Ser. A Mat. (RACSAM), 112(2018), 1157-1168.

[32] H. M. Srivastava, A. K. Mishra and P. Gochhayat, Certain subclasses of analytic and bi-univalent functions, Appl. Math. Lett., 23(2010), 1188-1192.

[33] H. M. Srivastava, A. Motamednezhad and E. A. Adegani, Faber polynomial coefficient estimates for bi-univalent functions defined by using differential subordination and a certain fractional derivative operator, Mathematics, 8(2020), Art. ID 172, 1-12.

[34] H. M. Srivastava, N. Raza, E. S. A. AbuJarad, G. Srivastava and M. H. AbuJarad, Fekete-Szegö inequality for classes of $(p, q)$-starlike and $(p, q)$-convex functions, Rev. Real Acad. Cienc. Exactas Fís. Natur. Ser. A Mat. (RACSAM), 113(2019), 3563-3584.

[35] H. M. Srivastava and A. K. Wanas, Initial Maclaurin coefficient bounds for new subclasses of analytic and m-fold symmetric bi-univalent functions defined by a linear combination, Kyungpook Math. J., 59(3)(2019), 493-503.

[36] S. R. Swamy, A. K. Wanas and Y. Sailaja, Some special families of holomorphic and Sălăgean type bi-univalent functions associated with (m,n)-Lucas polynomials, Communications in Mathematics and Applications, 11(4)(2020), 563-574. 
[37] P. Vellucci and AM. Bersani, The class of Lucas-Lehmer polynomials, Rend Math. Appl., 37(2016), 43-62.

[38] F. Yousef, T. Al-Hawary and G. Murugusundaramoorthy, Fekete-Szegö functional problems for some subclasses of bi-univalent functions defined by Frasin differential operator, Afr. Mat., 30(2019), 495-503.

[39] F. Yousef, B. Frasin and T. Al-Hawary, Fekete-Szegö inequality for analytic and bi-univalent functions subordinate to Chebyshev polynomials, Filomat, 32(2018), 3229-3236.

[40] T. Wang and W. Zhang, Some identities involving Fibonacci, Lucas polynomials and their applications, Bull Math. Soc. Sci. Math. Roum., 55(2012), 95-103.

[41] A. Zireh, E. Analouei Adegani and S. Bulut, Faber polynomial coefficient estimates for a comprehensive subclass of analytic bi-univalent functions defined by subordination, Bull. Belg. Math. Soc. Simon Stevin, 23(2016), 487-504. 